\title{
Preconception counseling for epileptic mothers treated with antiepileptic drugs
}

\author{
Andreea Elena Dumitru', Anca Marina Ciobanu', Radu Botezatu',2, Nicolae Gica ${ }^{1,2}$, \\ Corina Gica', Gheorghe Peltecu ${ }^{1,2}$, Anca Maria Panaitescu ${ }^{1,2}$ \\ ${ }^{1}$ Filantropia Clinical Hospital, Bucharest, Romania \\ 2"Carol Davila" University of Medicine and Pharmacy, Bucharest, Romania
}

\begin{abstract}
Planning a pregnancy for a woman diagnosed with epilepsy might be a challenge, but nowadays it is no more a contraindication. By offering careful education to this population, maternal and neonatal normal outcome is expected. It is important to have a multidisciplinary team of professionals that include a neurologist, a maternal fetal medicine specialist, an obstetrician and an anesthesiologist that manage the case and inform the women about potential risks for them and for fetuses, evolution of their scheme of treatment and particular adjustments that they need to do, as well as advices and strategies for postpartum period. The goal should always be optimal seizure control and minimal exposure to antiepileptic drugs for fetus.

Preconception counseling should accomplish the four most important steps for the benefits of both mother and fetus: particular choice of antiepileptic drugs and the lowest effective dose, valproate avoidance, folate supplementation and birth control with contraceptives until baseline is achieved.

Finally, although there is an increased risk for congenital malformations and neurodevelopment disorders, a good managed pregnancy can maintain to a minimum the fetal exposure to side effects of antiepileptic drugs and maternal risk for seizure.
\end{abstract}

Keywords: antiepileptic drugs, epilepsy, congenital malformations, neurodevelopment, preconception planning

\section{INTRODUCTION}

During pregnancy, maternal organism adapts to different physical, physiological and psychic changes that occur, for both benefits of mother and fetal development. Epilepsy is a condition that requires particular care, but nowadays it is no longer a contraindication to pregnancy, with more than $90 \%$ of women having good outcomes [1].

\section{DIAGNOSIS}

Should be made by a medical practitioner specialized in the field of neurology, taking into consideration that it is a heterogeneous group of diseases affecting the brain with most frequent manifestation-seizures. First assessment of this condition during pregnancy should include patient history, type, duration and frequency of seizures and treatment history regarding effectiveness of drugs administered. It is recommended that women under treatment with antiepileptic drugs (AEDs) that intend to get pregnant, to continue to use contraception until a discussion with a neurologist and an obstetrician, because the dosage and type of medication administered is easily and more secure changed in the pre partum period.

Reviewing the literature, it indicates that mortality and morbidity rates are increased among women diagnosed with epilepsy. Maternal mortality appears to be 10 times higher in pregnant women with epilepsy than the general population [2]. Possible explanations include an increase in medical 
comorbidities among this population, life threatening complications or seizure-related complications. SUDEP (sudden unexpected death in epilepsy) is also mentioned as possible cause for maternal mortality, with one third of deaths produced in the postpartum and two-thirds in women treated with lamotrigine [3].

\section{FIRST SEIZURE DURING PREGNANCY}

The primary target in evaluating a seizure is to determine whether it is caused by a treatable systemic process or by an intrinsic dysfunction of the CNS. The initial evaluation of a first seizure include a medical history provided by the patient or by relatives that can describe the event and exclude possible diagnostics, but also to provide information about family history and other comorbidities. Secondly, a description as accurate as possible of the seizure is needed, including circumstances of production, ictal behaviors, duration, severity and postictal state. For establishing a diagnosis, it is important to know prior evens that may have happened, seizure triggers and medical history of the patient, including any medication taken.

In case of pregnant woman, diagnostic considerations should exclude conditions such as eclampsia or cerebral venous thrombosis. Evaluation of neurological status should consider the stage of pregnancy, bearing in mind concerns about level of ionizing radiation on the fetus. Magnetic resonance imaging can be used at any stage of pregnancy, when information cannot be acquired by nonionizing procedures, avoiding use of gadolinium.

Treatment for a first seizure observed during pregnancy should avoid use of valproate, due to its linking to neurodevelopmental disorders and fetal malformations. It is recommended the use of levetiracetam, confirmed with a safety reproductive profile, a spectrum of action of multiple types of seizures and the possibility to start the therapeutic dose immediately. Although, effective during pregnancy, lamotrigine is not a desired option for starting during pregnancy because of difficulties such as risk of rash and enhanced clearance in order to titrate a therapeutic concentration [4].

\section{COMPLICATIONS}

Frequent complications of these pregnancies include: preeclampsia, preterm birth, placental abrup- tion, postpartum excessive bleeding, fetal growth restriction, stillbirth or fetal death in utero. It is still uncertain if the use of antiepileptic drugs may produce a higher risk of abortion in pregnant women with epilepsy compared to the others. On the other hand, the antiepileptic drugs exposure is associated with an increased risk for preterm birth [5].

\section{PRECOUNSELING}

Preconception counseling should be offered to all women of childbearing age diagnosed with epilepsy to minimize risks. Key issues regarding pregnancy and management of a case with epilepsy should be clearly explained by medical specialists as follows:

- Need for periodical visits to the neurologist to review the medical history, imaging and electro-encephalography (EEG) findings to confirm the diagnosis and if present, type of seizures

- Perform blood analyses for AED levels and discussion about change of dosage or medication during pregnancy

- Interaction with a multidisciplinary team: neurologist, obstetrician, maternal fetal medicine specialist.

Preconception planning should include information about birth control, need for AEDs and choice of type accordingly and importance of folic acid supplementation (Figure 1).

\section{INTRAPARTUM CARE}

During intrapartum period women become more susceptible to seizure triggers and may experience an increase in seizure occurrence. Common triggers include sleep deprivation, emotional stress, metabolic disorders caused by hyperemesis gravidarum.

\section{First trimester guidance}

It includes continuation of folic acid supplementation to prevent neural tube defects, adjustment and changes in treatment options and screening for malformations. Due to an increase in the volume of distribution, renal clearance and hepatic metabolism of AEDs, blood levels should be monitored monthly. Exceptions are made if patients report in- 


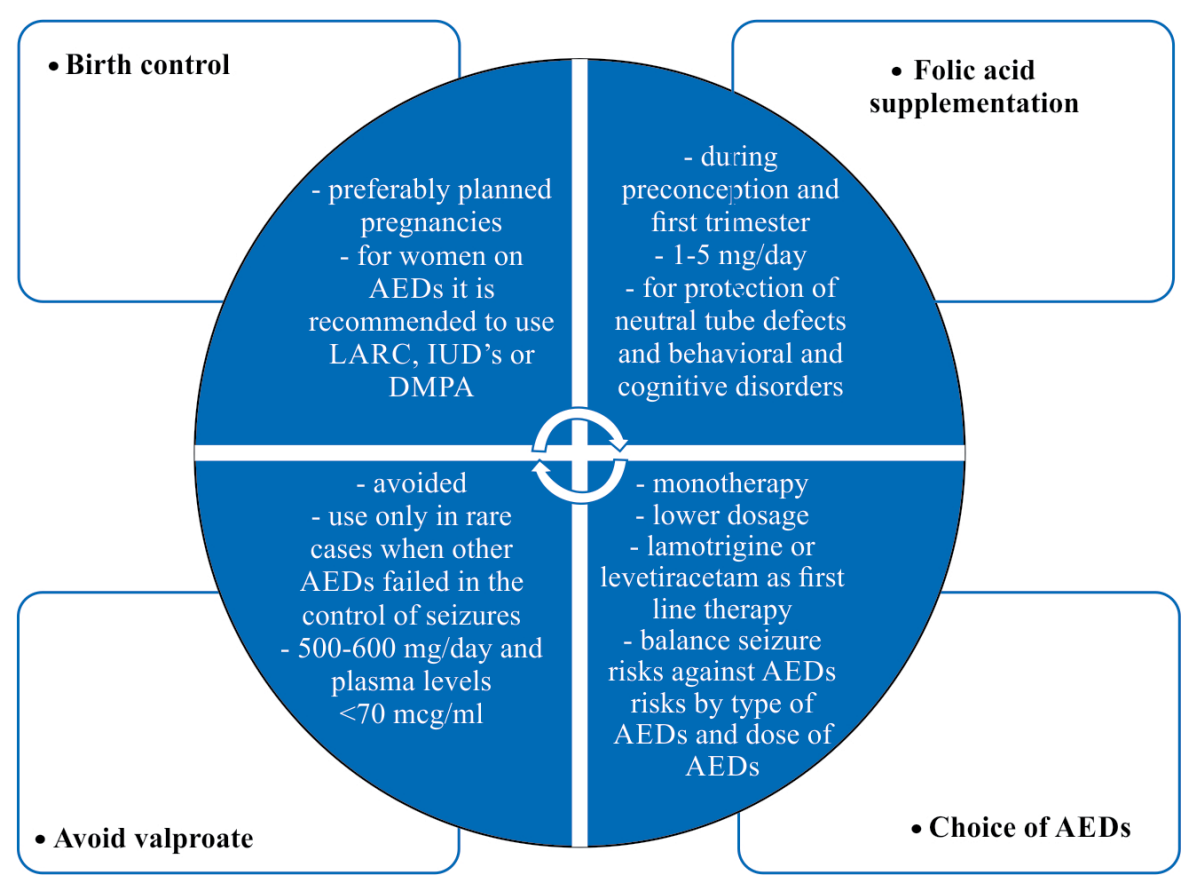

FIGURE 1. Preconception planning

creased seizure activity or worsened symptoms like dizziness, blurred vision or others associated with AEDs toxicity, when monitoring should be performed immediately.

There is a category of AEDs that are highly protein bound (eg, phenytoin, phenobarbital, valproate, carbamazepine) and the total plasma drug level may decrease with impaired protein binding, but the physiologically important free or unbound drug concentration may not change as much, while the most pronounced increases in AEDs clearance (corresponding to serum level decrease) are associated with lamotrigine and levetiracetam [2].

Another suggestion for first trimester therapeutical conduct is to re-dose AEDs if emesis occurs shortly after AEDs intake, in order to maintan the baseline.

Regarding change of AEDs, it showed no effectiveness as means of teratogenic effects avoidance with more potential risks to seizure occurrence. As far as the potential teratogen exists during the first nine weeks, significant exposure has already occurred and alteration of the actual scheme of treatmentmay provides more harm than benefits.

Moreover, first trimester recommendations include screening for malformations. Epilepsy does not chnage a woman's risk of chromosomal aneuploidy. As for general women, screening for aneuploidy is based on mother's age at the time of ex- pected delivery, ultrasound findings and paraclinical analisis (beta hCG and PAPP-A). If the result is uncertain, measurement of the serum alpha-fetoprotein (AFP) concentration or amniocentesis for amniotic fluid AFP levels should be performed between at or after 16 weeks, especially in women treated with valproate and carbamazepine $[2,6,7]$.

Usually, for the patient's benefits, the treatment options should not be changed, but as stated from a 2019 report of ILAE, there are a few situations in which it is permitted to increase the AEDs dose after the first trimeter, when AEDs blood levels cannot be measured. The following situations are [8]:

- Use of AEDs prone to important changes in clearance during pregnancy ( lamotrigine, levetiracetam)

- Focal seizures or generalised tonic-clonic ones

- Management of seizure was sensitive to changes in AEDs levels before pregnancy

- Lowest dose of AEDs at the beginning of pregnancy

\section{Second trimester guidance}

Advocate for continuous monitoring of AEDs levels monthly, adjust doses to maintain baseline and in case of seizures or side effects, combining medical history and neurological examination, re- 
view results from prenatal tests and make a screening for anxiety and depression.

\section{Third trimester guidance}

It must take into account the possible worsening of symptoms, especially increased risk for seizures during peripartum period. The multidisciplinary team should advise the women involved and recommend a birth plan accordingly. Breastfeeding should remain an opened option also for this population, with clear explanations from medical personnel and evidenced based data that benefits outweigh risks. During the postpartum phase, doctors and patient should establish some strategies that may include a third person for help, while the recent mother can have some sleep and rest at least $4 \mathrm{~h}$ uninterrupted a night. Being a period with emotional implications, stress and many risk factors, screening for depression and anxiety should be assessed frequently. Regarding medication, using a determination of AEDs levels at 34 and 37 weeks, a postpartum taper plan must be realized. Last, but not least, the newborn safety should be assured permanently, by monitoring signs for adequate hydration and nutrition.

\section{DELIVERY}

The birth plan for a woman diagnosed with epilepsy should consider both recommendation from obstetrician, maternal fetal medicine specialist and neurologist. AEDs doses must be obtained during labor. As certified in Kerala registry of epilepsy and pregnancy, the peripartum period has a higher risk for seizures, especially the three days: the one prior to delivery, the day of delivery and the day after [9]. Most of this category can have a normal vaginal delivery, but pain management and labor should be discussed with an anesthesiologist prior to delivery. Neuraxial analgesia is encouraged and a proper epidural may help the woman to rest during first stage of labor and minimize the risks of sleep deprivation and stress. One member of the family may join the patient during labor and lights can be lowered to offer more comfort.

In case of a convulsive seizure during labor, it should be treated promptly with benzodiazepines, lorazepam being the drug of choice, $1 \mathrm{mg}$ iv for non-convulsive seizure and $2 \mathrm{mg}$ iv for generalized tonic-clonic ones. Continuous fetal monitoring is advisable because fetal tracing might be altered by maternal seizure, but it should return to normal in 5 minutes. If not, given the risk for placental abruption during a crisis, a surgical intervention might be the most appropriate solution. When a seizure is first produced during third trimester or early postpartum period it is difficult to distinguish it from eclampsia and both can be treated until one can be excluded [15].

As regards neonatal safety, medication such as benzodiazepines and phenobarbital remain in newborn plasma for several days causing sedation and hyporesponsiveness, with a gentle effect if monotherapy is used.

\section{EFFECTS ON NEWBORNS AND INFANTS}

In women with epilepsy, neonatal care involves a few safety precautions:

- the mother is encouraged to have regular intervals for sleeping and resting

- the mother should be discouraged to bathe herself behind a closed or even locked door

- driving and sleeping with the baby in the same bed should be avoided

- in case of a patient having myoclonic seizures, a sling might be used when walking with the baby

Regarding babies from mothers treated with AEDs there is an increased risk up to 4 to $6 \%$ for major congenital malformations, fetal growth restrictions and neurodevelopment disorders [10]. Particular types of malformations involved are neural tube defects, congenital heart defects, urinary tract defects, skeletal abnormalities and cleft palate $[2,11,12,13]$. Different medication has different effect on the fetus, with medical evidence that exposure is dose dependent. The figure below shows the most frequent medications used for treatment and their influence [2] (Figure 2).

There is elevated risk for growth restriction and preterm birth for children exposed to AEDs in utero. Studies show that small for gestational age is more common correlated with the use of valproate (14.5\%) and carbamazepine (12.9\%), even higher percentages for the use of same drugs produces microcephaly and low Apgar scores, under 7 [14].

The neurocognitive development of children is also influenced by the drugs and doses used during 
pregnancy, with negative impact of verbal and non-verbal ability, memory and executive functions. Independent predictors of a child's IQ were AEDs type and dose, maternal age and use of folate.

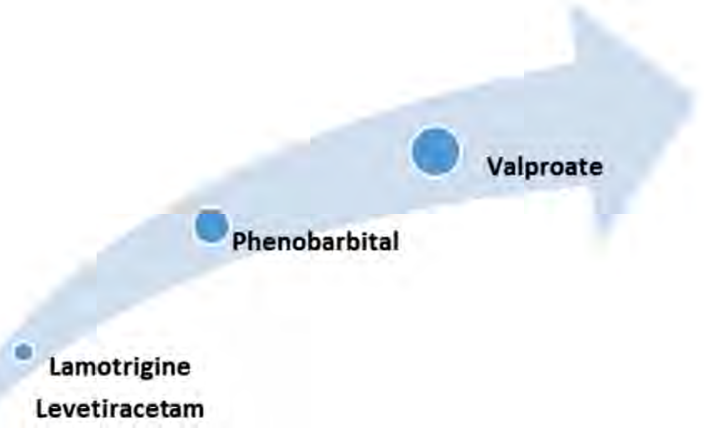

FIGURE 2. Risk for congenital malformations with different therapeutic options

\section{POSTPARTUM FOLLOW-UP}

Some indication for follow up in the postpartum period (6 weeks after birth) include review of medical history, seizures and drug's side effects, assessment for syndrome of maternal depression and fetal neurologic development. Both neurologist and obstetrician should provide a build-up short and long term plan for contraception, as well as a discussion regarding future pregnancies and timing.

\section{REFERENCES}

1. Harden CL, Hopp J, Ting TY, et al. Practice parameter update: Management issues for women with epilepsy - focus on pregnancy (an evidence-based review). Obstetrical complications and change in seizure frequency: Report of the Quality Standards Subcommittee and Therapeutics and Technology Assessment Subcommittee of the American Academy of Neurology and American Epilepsy Society. Neurology. 2009;73(2):126-132.

2. Tomson T, Battino D, Bromley R, et al. Management of epilepsy in pregnancy: a report from the International League Against Epilepsy Task Force on Women and Pregnancy. Epileptic Disord. 2019;21(6):497-517.

3. Edey S, Moran N, Nashef L. SUDEP and epilepsy-related mortality in pregnancy. Epilepsia. 2014;55(7):e72-e74.

4. Meador KJ, Baker GA, Browning N, et al. Fetal antiepileptic drug exposure and cognitive outcomes at age 6 years (NEAD study): A prospective observational study. Lancet Neurol. 2013;12(3):244-252.

5. Hernández-Díaz S, McElrath TF, Pennell PB, et al. Fetal growth and premature delivery in pregnant women on antiepileptic drugs. Annals of Neurology. 2017;82(3):457-465.

6. Lindhout D, Meinardi H, Meijer JW, Nau H. Antiepileptic drugs and teratogenesis in two consecutive cohorts: changes in prescription policy paralleled by changes in pattern of malformations. Neurology. 1992;42(4 Suppl 5):94-110.

7. Hobbins JC. Diagnosis and management of neural-tube defects today. N Engl J Med. 1991; 324:690.

8. Nadel AS, Green JK, Holmes LB, Frigoletto FD Jr, Benacerraf BR. Absence of need for amniocentesis in patients with elevated levels of

\section{CONTRACEPTION}

Women taking hormonal contraceptives have an increased risk for unplanned pregnancies if they use enzyme induces AEDs. It is because hepatic enzyme induction accelerates the metabolism and alters protein binding of estrogen and progesterone which may lower the efficacy of coadministered estrogen-progestin contraceptives and progestin-only contraceptives. Therefore it is recommened for this population the use of long-acting reversible contraceptive (LARC) choices of intrauterine devices (IUDs) or intramuscular depot medroxyprogesterone acetate (DMPA) [15].

\section{CONCLUSIONS}

Management of epilepsy during pregnancy might be challenging, but it is not a contraindication. Physiological modifications that occur during pregnancy may alter the pharmacokinetic of AEDs generating a higher risk for seizures and involving a more frequent blood dosage and dose adjustment. All in all, with suitable counseling prior to conception and careful education of patients by a multidisciplinary team, maternal and neonatal outcomes may be similar as in general population.

Conflict of interest: none declared Financial support: none declared

maternal serum alpha-fetoprotein and normal ultrasonographic examinations. N Engl J Med.1990;323(9):557-561.

9. Thomas SV, Indrani L, Devi GC, et al. Pregnancy in women with epilepsy : preliminary results of Kerala registry of epilepsy and pregnancy. Neurol India. 2001;49(1):60-66.

10. Sabers A, aRogvi-Hansen B, Dam M, et al. Pregnancy and epilepsy: a retrospective study of 151 pregnancies. Acta Neurol Scand. 1998;97:164.

11. Meador KJ, Pennell PB, Harden CL, et al. Pregnancy registries in epilepsy: a consensus statement on health outcomes. Neurology. 2008;71(14):1109-1117.

12. Hernández-Díaz S, Werler MM, Walker AM, Mitchell AA. Folic acid antagonists during pregnancy and the risk of birth defects. N Engl $J$ Med. 2000;343(22):1608-1614.

13. Tomson T, Battino D, Bonizzoni E, et al. Dose-dependent risk of malformations with antiepileptic drugs: An analysis of data from the EURAP epilepsy and pregnancy registry. Lancet Neurol. 2011;10(7):609-617.

14. Pennell PB, Klein AM, Browning N, et al. Differential effects of antiepileptic drugs on neonatal outcomes. Epilepsy Behav. 2012;24(4):449-456.

15. Pennell PB, McElrath T. Management of epilepsy during preconception, pregnancy, and the postpartum period. Available at https://www. uptodate.com/contents/management-of-epilepsy-during-preconception-pregnancy-and-the-postpartum-period. 